\title{
COMPLEXITÉ DES PHÉNOMĖNES DE COUPS DE BÉLIER SUR LES INSTALLATIONS DE POMPAGE ET ESSAI DE CLASSIFICATION DES SOLUTIONS GÉNÉRALES POUR Y REMÉDIER
}

\author{
THE COMPLEXITY OF WATER-HAMMER PHENOMENA IN PUMP INSTALLATIONS \\ AND AN ENDEAVOUR TO CLASSIFY THE GENERAL REMEDIAL SOLUTIONS \\ English synopsis p. 672
}

par Paul BERGERON

Ingénieur des Arts et Manufactures.

Les phénomènes dus aux coups de bélier à l'arrêt des pompes présentent, au point de vue hydraulique, une complexité bien plus grande que pour les turbines. Les raisons principales en sont les suivantes:

alors que sur les turbines le problème consiste à arrêter une colonne d'cau descendante, sur les pompes, le phénomène commence par le ralentissement d'une colonne d'eau ascendante;

dans la plupart des installations, l'entrainement des pompes se faisant par moteur électrique, il faut normalement considérer le cas de la disparition instantanée du couple moteur, due à la disjonction ;

une deuxième conséquence de la disjonction est que normalement les appareils de protection ne peuvent être commandés électriquement ;

enfin, il arrive bien souvent que le tracé des conduites soit quelconque. Suivant en général le relief du terrain, il présente une succession de points hauts, de points bas et des pentes variées.

Parallèlement à la complexité des phénomènes, il existe des procédés très divers et très différents pour y remédier. Le choix que I'on peut en faire apparaît souvent arbitraire surtout à l'usager. En fait, chaque procédé correspond à des cas bien déterminés et à une zone d'utilisation pratique.

Aucun dispositif n'est universel.

C'est la définition et un essai de classement des différents procédés que nous allons tenter d'établir.
D'une manière générale, les phénomènes provoqués par l'arrêt d'une pompe peuvent être décomposés en deux phases dont les conséquences sont nettement différentes:

La première phase est celle pendant laquelle la colonne d'eau propulsée en marche normale de bas en haut s'arrête, créant au départ de la pompe des ondes de dépression se propageant jusqu'aux réservoirs. Suivant le profil de la conduite, ces ondes de dépression risquent de créer des cavitations entraînant des ruptures de veine. Si durant cette première phase la cavitation n'est en général pas dangereuse, par contre, au cours de la deuxième phase, la fermeture des cavités peut être la cause de surpressions catastrophiques. Il faut donc dans toute la limite du possible empêcher la cavitation.

La deuxième phase est celle pendant laquelle la colonne d'eau s'étant arrêtée, inverse son sens d'écoulement sous l'effet de la dépression existant alors dans la conduite. Le courant inversé tend, d'abord à rétablir la pression normale dans la conduite, puis arrivant à la station à faire apparaitre des surpressions dangereuses qu'il faudra limiter.

Ce n'est qu'au moment où, le courant étant inversé, la pression redevient égale ou supérieure à la pression normale que le problème s'apparente à celui de l'arrêt sur turbine.

Pour une classification rationnelle des procédés, il y a lieu de considérer tout d'abord ceux destinés à limiter les dépressions, car ce sont ceux dont les conditions d'utilisation sont fonc- 
tion des conditions d'installation, en porticulier longueur et tracé des conduites, vitesse initiale, etc...

Viennent ensuite les procédés destinés à limiter les surpressions qui dépendent ou même dans certains cas sont confondus avec les précédents.

Pour simplifier l'exposé qui va suivre, il ne sera considéré que le cas de conduite unique ovec station de pompage à l'origine et réservoir au refoulement. Les cas complexes peuvent entrainer évidemment la combinaison des différents procédés.

\section{1. - PROCEDES POUR LIMITER LES DEPRESSIONS}

En supposant un arrêt brusque des groupes annulant instantanément le débit, la dépression a Vo

serait $-\frac{\mathrm{V}}{\mathrm{g}}$

Sauf dans les cas de pompe à très grande hauteur, ou lorsque la vitesse dans les conduites est excessivement faible, cette dépression crée presque toujours. le vide dans toute la conduite ou tout au moins dans une fraction de la conduite.

Elle est donc normalement excessive et devra être limitée à des valeurs acceptables qui, autant que possible, devront éviter la cavitation en tous les points de la conduite.

Les procédés utilisés dans ce but peuvent être classés comme suit :

\section{Première classe.}

Principe: empêcher l'arrêt instantané de la pompe.

Le couple du moteur cessant brusquement, seule l'inertie du groupe peut limiter l'instantanéité de l'arrêt.

La solution consiste donc à augmenter cette inertie par un volant.

L'inertie du groupe est toujours une condition favorable, car elle évite l'onde raide. Mais si le volant doit à lui seul parer à la cavitation dons toute la conduite, son dimensionnement devra être tel, qu'après un aller et retour d'onde, c'est-à-dire un temps proportionnel à la longueur de la conduite, la vitesse du groupe soit encore suffisamment grande pour limiter la dépression à des valeurs compatibles avec le tracé de la conduite.

C'est ce que met en évidence immédiatement la méthode graphique. Pour limiter la dépression maxima à une certaine valeur $M N$ (fig. 1),

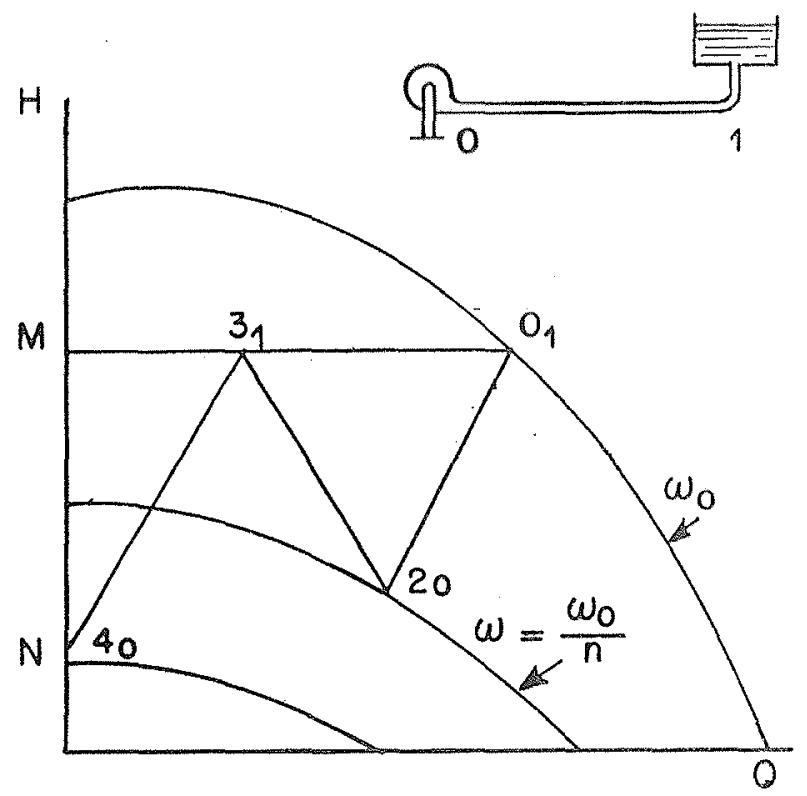

Fig. I

il faudra qu'après le temps $\Delta t$ d'un aller et retour d'onde à partir de la disjonction, la vitesse du groupe $(1)=\frac{\left.{ }^{(1)}\right)}{n}$ soit telle que le fonctionnement soit en un point 2 , de pression certainement supérieure à $\mathrm{N}$.

$\mathrm{Si}$, pour simplifier le raisonnement, il est supposé que le couple hydraulique de la pompe varie comme le carré de la vitesse angulaire, c'est-à-dire:

$$
\frac{C}{\omega^{2}}=\frac{C_{0}}{\omega_{0}{ }^{2}}
$$

où $C_{0}$ est le couple au point $O$ à la vitesse $\omega_{0}$ et $C$ le couple à la vitesse $\omega$, comme $\frac{C}{1}=\frac{d \omega}{d t}$, avec I moment d'inertie du groupe, on trouve que le temps $\Delta t$ qui s'écoule pour passer de la vitesse (1) ${ }_{0}$ à la vitesse $(1)=\frac{(1))}{n}$ est : (1) $\Delta t=1 \frac{\omega_{01}{ }^{2}}{P_{0}}(n-1)$, où $P_{0}=C_{01} \omega_{n}$ est la puissance du groupe.

I est la somme de $I_{M}$ inertie du moteur (celle de la pompe est toujours très faible) et de $I_{v}$ inertie du volant éventuellement ajouté.

En ce qui concerne l'inertie $I_{M}$ des moteurs en fonction des puissances et des vitesses, nous 
avons tiré des documents communiqués par un constructeur pour sa série courante:

$$
I_{M}=A \frac{P_{0}^{1,33}}{\omega_{0} 1,73},
$$

ce qui donne pour le terme $I_{M} \frac{{ }^{(1)^{2}}}{P_{0}}$ de la formule (l) A $P_{0}{ }^{0,33} \quad m_{0} 0,26$, donc variant peu ovec $P_{0}$ et $\omega_{0}$

De même pour l'inertie $I_{v}$ du volant additionnel, les conditions de vitesse périphérique, de limitation de la perte par frottement sur l'air en pourcentas 2 de la puissance totale, de poids et de prix, bien que difficiles à mettre en équation, font que pratiquement le terme $I_{v} \frac{\omega_{0}{ }^{2}}{P_{j}}$ de la formue (I), qui tend malgré tout à croitre avec $P_{0}$, ne varie que dans des proportions relativement restreintes malgré des variations de puissance de l'ordre de 100, 500 ou même plus.

En conclusion, le temps $\Delta t$ nécessaire pour passer de la vitesse initiale d̀ une vitesse $n$ fois plus petite atteindra des valeurs, pour des iner.. ties maxima normales, qui varieront relativement peu pour de grandes variations de puis.sance et de vitesse des groupes.

Comme $\Delta t$ représente dans le raisonnement actuel te temps d'un aller et retour d'onde, il définit la longueur de la conduite dont les valeurs maxima vont être limitées de ce fait dans tous les cas.

Malgré l'approximation du raisonnement précédent et bien que la valeur de ri varie beaucoup d'une installation à l'autre en fonction des caractéristiques de la pompe, du réseau, des caractéristiques et du tracé des conduites, il est possible d'indiquer que le volaní employé comme seul dispositif de protection ne pourra convenir que sur des conduites relativement courtes. En dehors des cas particuliers pour lesquels on accepte de sacrifier le prix ou le rendement, la zone d'utilisation pratique est pour des conduires de quelques centaines de mètres. De toute manière, pour 1.000 mètres ef même moins on ne peut à priori envisager la solution par volant comme certainement possible ou tout au moins économiquement admissible.

\section{Deuxième classe.}

Principe : au départ de la station, substituer au débit de la pompe, un débit d'eau, soit que cette eau ait été mise préalablement en réserve (réservoir à air et cheminée d'équilibre), soit qu'elle soit prélevée directement sur l'aspiration des pompes.

\section{A) RESERVOIR A AIR.}

L'application de ce dispositif n'est limitée que par des considérations économiques ou par des considérations d'exploitation.

En principe, rien ne s'oppose à prévoir un réservoir à air dans tous les cas si ce n'est son prix lorsque les dimensions et les pressions deviennent trop importantes.

Dans I'hypothèse très simplificatrice où les pertes de charge sont considérées comme nulles et où on néglige la variation du niveau du plan d'eau dans le réservoir pendant la détente, le volume $V$ utile du réservoir s'exprime par la formule:

$$
v=\frac{\omega}{2 g} \frac{L Q_{0} U_{0}}{P_{0}\left[1-\frac{p}{P_{0}}+\frac{P}{P_{0}} \log \frac{p}{D_{0}}\right]}
$$

cù :

$\checkmark$ : volume maximum occupé par l'air à la fin de la détente;

$L$ : longueur de la conduite;

$Q_{0}$ : débit initial;

$\mathrm{U}_{0}$ : vitesse initiale dans la conduite;

$\mathrm{p}_{0}$ : pression absolue initiale de l'air du réservoir ;

$p$ : pression absolue de l'air à la fin de la détente.

Cette pression $\mathrm{p}$ minimum est conditionnée par le profil de la conduite et doit être choisie pour qu'en aucun point il n'y ait cavitation.

Bien que très élémentaire, cette équation permet de constater que pour un $\frac{\mathrm{p}}{\mathrm{p}_{0}}$ donné le volume du réservoir est proportionnel à $\frac{L Q_{0} U_{0}}{p_{0}}$.

Comme $U_{0}$ vitesse économique varie en général peu d'une installation à l'autre, le volume du réservoir pour un même rapport de la pression fincle à la pression initiale est finalement proportionnel à :

$$
L Q_{0}
$$

$\mathrm{P}_{0}$

c'est-à-dire que, pour les dimensions économiquement acceptables, le réservoir constituera 
une solution intéressante pour de faibles débits et de grandes longueurs de conduites ou inversement pour des débits plus importants et des conduites courtes.

Pour donner des ordres de grandeur chiffrés, un réservoir déjà important de 4 à $6 \mathrm{~m}^{3}$ permet. tra, pour une pression de marche moyenne, de limiter la dépression à des valeurs acceptables sur une station de $1 \mathrm{~m}^{3} / \mathrm{s}$ refoulant à une distance de l'ordre du $\mathrm{Km}$, ou sur une station de $0,1 \mathrm{~m}^{3} / \mathrm{s}$ refoulant à une distance de l'ordre d'une dizaine de $\mathrm{Km}$.

Quant à la pression $p_{0}$ initiale qui est favorable à la limitation des dimensions du réservoir, elle conduit par contre à l'augmentation des épaisseurs, et comme vers 10 à $15 \mathrm{Kgs}$ la question de dissolution de l'air dans l'eau devient importante, il est bien rare de voir dans les applications courantes ces valeurs dépassées.

\section{En résumé :}

En dehors des cas où des conditions d'exploitation très particulières rendent intéressanłs des réservoirs de plusieurs dizaines de $\mathrm{m}^{3}$, les réservoirs à air sont surtout employés sur des installations de faibles débits $\left(1 \mathrm{~m}^{3} / \mathrm{s}\right.$ paraissant déjà une limite maximum peu fréquente), pour des distances variant en raison inverse des débits, et pour des pressions inférieures à $15 \mathrm{Kgs}$.

A noter en outre que le réservoir à air nécessite une surveillance permanente, le manque d'air provoquant obligatoirement l'accident.

\section{B) CHEMINEE D'EQUILIBRE}

\section{ET RESERVOIR SURELEVE.}

Lorsque les hauteurs géométriques sont faibles et lorsque les lieux le permettent, la cheminée d'équilibre ou le réservoir surélevé au départ de l'usine, jouent le même rôle que le réservoir à air, autorisent des débits beaucoup plus importants, et ne nécessitent aucune surveillance.

Mais sur les stations de pompage il est très rare d'avoir les caractéristiques et les conditions favorables pour l'adaptation de ces dispositifs. En outre, il faut considérer que le réservoir est toujours coûteux.

\section{C) RESERVOIR A EAU.}

La station étant normalement un point bas de la canalisation de refoulement, on ne peut envisager la solution avec soupape laissant entrer l'air au moment de la dépression, cet air risquant de remonter le long de la conduite. Par contre, il est possible d'envisager un réservoir normalement plein d'eau en charge sur la con- duite et muni à sa partie supérieure d'une soupape s'ouvrant à la dépression. A l'arrêt du groupe, ce réservoir se vide dans la conduite en maintenant la pression atmosphérique plus la charge au départ de la conduite de refoulement.

11 joue ainsi le rôle d'une cheminée d'équili. bre, mais dans laquelle le niveau serait inférieur à celui du réservoir de refoulement.

Ce dispositif est intermédiaire entre la cheminée d'équilibre et le dispositif décrit ci-dessous au $\S$ a de $D$. II ne peut s'appliquer que dans des. cas où le tracé de la conduite de refoulement ne s'élève que de quelques mètres au-dessus du réservoir; d'autre part, comme nous le verrons plus loin, la soupape doit se fermer lentement au retour de la pression, si bien que ce dispositif est d'un emploi relativement rare.

\section{D) ALIMENTATION DE LA TUYAUTERIE DE REFOULEMENT PAR L'ASPIRATION.}

Deux cas sont à considérer :

a) La longueur de la tuyauterie d'aspiration est négligeable devant celle de refoulement.

Si la hauteur d'élévation et le tracé de la conduite de refoulement sont tels, que le fait de maintenir au départ de la station une cote piézométrique voisine de celle de l'aspiration, évite la cavitation de la conduite de refoulement, il suffit, dans ce cas assez particulier, de prévoir sur le refoulement un piquage, muni d'un clapet, en communication avec l'aspiration (fig. 2).

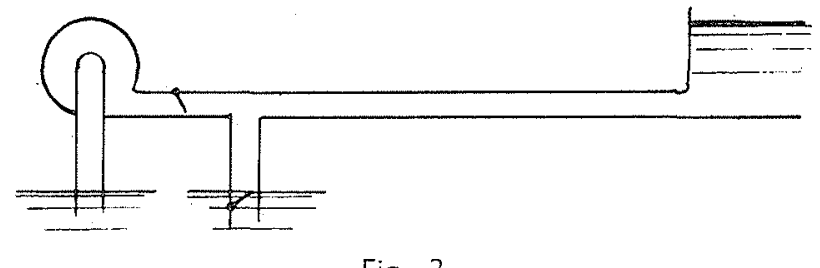

Fig. 2

Quand la pression au refoulement, au moment de l'arrêt, atteint la pression d'aspiration, le clapet se soulève et l'eau passe directement de I'aspiration au refoulement.

A remarquer que le principe de ce dispositif est le même que celui de la cheminée d'équilibre. Mais dans ce cas le volume en est infini et le niveau est celui de l'aspiration. II ne trouve son application que lorsque la tuyauterie de refoulement a un tracé s'élevant de quelques mètres seulement au-dessus du niveau d'aspiration.

b) La longueur de la tuyauterie d'aspiration n'est pas négligeable par rapport à celle de la ruyauterie de refoulement. 
II faut en outre que la tuyauterie d'aspiration puisse supporter une surpression.

L'arrêt de la pompe qui crée initialement une dépression au refoulement, crée par contre une surpression d̀ l'aspiration puisque tout se passe comme si brusquement la tuyauterie d'aspiration était étranglée.

A la disjonction la pression augmente donc à l'aspiration, tandis qu'elle diminuera au refoulement. En utilisant la méthode graphique (fig. 3), à l'origine des temps, si on néglige les

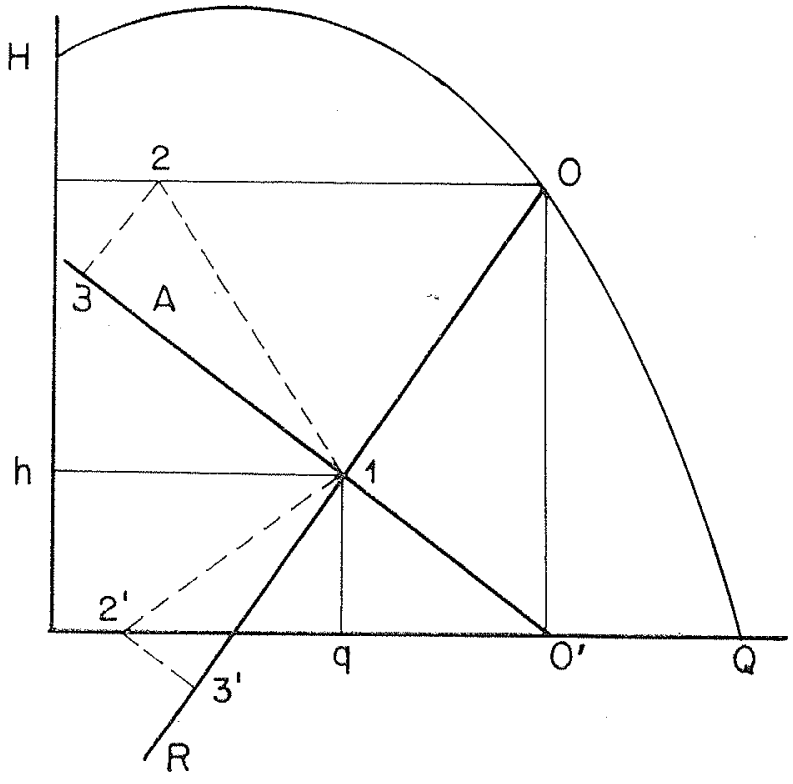

Fig. 3

pertes de charge, la pression au refoulement variera suivant $O R$ de coefficient angulaire $+\frac{a}{g S_{r}}$, la pression à l'aspiration suivant $\mathrm{O}^{\prime} \mathrm{A}$ de coefficient angulaire $-\frac{a}{g S_{a}}$ où $S_{i}$ et $S_{a}$ sont respectivement les sections de la tuyauterie de refoulement et de la tuyauterie d'aspiration.

Au point 1, où les pressions seront égales, et tendent à s'inverser, si un clapet est prévu sur un by-pass de la pompe (fig. $3^{\prime}$ ), il se soulèvera et le débit $g$ passera de l'aspiration au refoulement maintenant en ce point la pression à la valeur h. Puis l'épure continue.

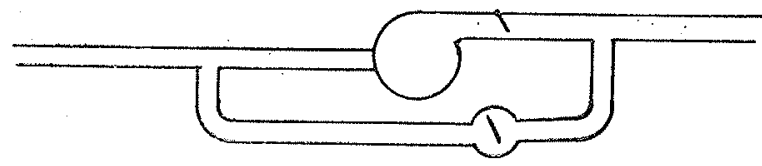

Fig. $3^{\prime}$
Si la conduite d'aspiration est plus lonque que la tuyauterie de refoulement, les pressions à la pompe continueront à se déplacer sur la droite O'A soit 1 - 3 etc... pendant le temps d'un aller-retour d'onde de la pompe au réservoir d'aspiration; dans le cas contraire les pressions se déplaceront sur la droite $\mathrm{OR}$, soit $1-3^{\prime}$ etc..., pendant le temps d'un aller-retour d'onde de la pompe au réservoir de refoulement. Théoriquement dans l'hypothèse où les conduites d'aspiration et de refoulement ont même longueur et même section et en supposant nulles les pertes de charge, la pression à la pompe se maintient constante et égale à la moirié de la hauteur d'élévation.

On peut ainsi dans certains cas, et en particulier lorsque la huyauterie d'aspiration est plus longue que la ruyauterie de refoulement, maintenir au départ de la conduite de refoulement, pendant un temps suffisant, une pression netiement supérieure à celle correspondant au riveau d'aspiration ef éviter de ce fait la cavitation.

A noter que si la pression obtenue ainsi au départ de l'usine est surabondante pour éviter la cavitation dans la conduite de refoulement, il est possible de supprimer le by-pass et de laisser simplement le courant d'eau s'établir à travers la pompe. Dans ce cas, dès que la pompe, en se ralentissant sous l'effet de son inertie, cesse de faire une pression positive, elle fonctionne en turbine centrifuge, et crée une chute de pression entre son entrée et sa sortie, qui diminue d'autant la pression au départ de la tuyauterie de refoulement.

\section{Troisième classe}

Principe: les dispositifs précédents prévus au départ de la station ne permettant pas d'éviter la rupture de la veine liquide ou refoulement, fractionner la conduite en éléments comportant chacun un dispositif de protection particulier.

C'est ce qui se présente pour les très gros débits, les conduites très longues, ou ayant sur leur tracé des points hauts à une altitude voisine de celle du réservoir de refoulement.

Le tracé le plus général d'une conduite comportant des points bas et des points hauts, les dispositifs de protection se trouveront normalement aux points hauts où risque de se produire la cavitation.

A noter que dans ce cas de fractionnement, bien que les ondes qui parcourent les différents tronçons interfèrent les unes sur les autres, le premier tronçon qui part de la station de pompage, et qui se trouve réduit à des longueurs 
limitées du fait du fractionnement, devra être équipé pour sa propre part d'un des dispositifs déjà envisagés.

Pour les autres troncons, les dispositifs utilisés peuvent se classer comme suit:

A) ALIMENTER LES POINTS HAUTS OU SE PRODUIRAIT LA CAVITATION PAR DE L'EAU MISE EN RESERVE.

Les dispositifs sont les mêmes que ceux déjà examinés au départ de la station de pompage. II y a lieu de faire toutefois les remarques suivantes

a) Le réservoir à air qui peut convenir lorsque les pressions de marche au point haut considéré sont encore élevées, n'est pratiquement pas utilisé parce que trop éloigné de l'usine alors qu'il nécessite une surveillance et une réalimentation fiéquente en air.

b) La cheminée d'équilibre et, à sa limite, le reservoir surélevé, trouvent avantageusement leur application lorsque le terrain le permet dans le cas d'un tracé analogue à celui rencontré fréquemment sur les installations de turbines, c'est-à-dire avec montée rapide au départ de l'usine puis longue galerie ou tuyauterie horizontale sous faible charge.

c) Le réservoir à eau n'est à préférer à la scupape, examinée plus loin, que dans la limite où il est dangereux de laisser pénétrer de l'air dans la conduite parce qu'il s'agit d'un point d'inflexion et non d'un point haut proprement cit, ou encore lorsque, séparé de la conduite par un clapet percé qui se ferme dès que la pression réapparaît, il permet de supprimer le dispositif mécanique de fermeture lente de la soupape. Encore faut-il dans ce dernier cas que, par suite de toutes les autres conditions, la fermeture brusque du clapet ne provoque pas de surpression dangereuse. Son emploi en est alors délicat.

B) LAISSER ENTRER L'AIR DANS LA CONDUITE DES QUE LA DEPRESSION APPARAIT.

S'il s'agit d'un point haut caractérisé, on prévoit une soupape s'ouvrant à la dépression et maintenant la pression atmosphérique au point où elle se trouve.

La veine liquide se rompt, chaque tronçon liquide, en amont et en aval de la soupape, évoluant pour son propre compte en fonction des conditions existant à leurs extrémités. La cavité cinsi formée se remplit d'air qui se localise au point hout.

Lorsque les courants s'inversent la poche se referme en chassant l'air par la soupape. Au moment de la fermeture de la poche, c'est-àdire lorsque les deux tronçons de la veine précédemment rompus reviennent en contact, afin d'éviter une surpression qui peut être supérieure a $\mathrm{Vo}$

à calculée avec la vitesse initiale, il faut 9

que la soupape soit encore ouverte puis qu'elle se ferme lentement dès que la pression réapparait, suivant une loi à déterminer dans chaque cas.

Un certain volume d'eau est obligatoirement rejeté hors de la conduite. Ce dispositif peut être assez généralisé mais présente l'inconvénient d'être mécanique et de nécessiter un entretien alors que par définition il est éloigné de l'usine.

\section{C) ACCEPTER LA CAVITATION.}

Comme dans le cas précédent, la veine liquide se rompt, mais au lieu d'ouvrir la poche à l'atmosphère, on laisse s'établir le vide absolu, ce qui ne présente aucun inconvénient au cours de la première phase si la conduite peut résister au vide.

Mais il faut éviter les surpressions pouvant résulter de la fermeture brutale des poches de vide. A cet effet, il y a lieu de disposer à des emplacements judicieux de la conduite et, en principe, sur chaque élément tronçonné, des clapets percés d'un orifice tel que lorsque la veine revient en arrière la surpression au clapet, qui se ferme brusquement, soit limitée à des valeurs acceptables et que d'autre part le débit qui passe par le trou du clapet fermé soit suffisamment faible pour que son arrêt brusque au moment de la fermeture de la poche ne provoque qu'une surpression limitée.

Ce dispositif qui ne peut guère être employé que sur des conduites de diamètre faible ou moyen présente l'avantage d'être sommaire et sans surveillance, mais implique des conditions particulières pour réaliser les deux conditions ci-dessus.

Parallèlement au dispositif de la soupape s'ouvrant à la dépression on pourrait envisager dans le cas actuel une soupape, disposée au point haut, restant fermée au vide, et s'ouvrant à la surpression au moment précis de la fermeture de la poche. Elle devrait s'ouvrir instantanément c) la surpression et avoir une caractéristique de hauteurs en fonction des débits de fuite régulièrement descendante jusqu'au débit nul pour lequel la hauteur serait légèrement supérieure à la charge normale. 


\section{II. - PROCEDES POUR LIMITER LES SURPRESSIONS}

Quand il y a eu rupture de veine, lorsque après l'inversion de courant la poche se referme s'il n'a pas été pris de précautions particulières, nous avons déjà dit que la surpression pouvait atteindre des valeurs dépassant même parfois de a Vo

beaucoup le _alculé avec la vitesse initiale 9

dans la conduite. Mais même dans le cas où il n'y a pas eu rupture de veine, grâce aux dispositifs adoptés pour la limitation de la dépression, lorsque l'eau, s'étant arrêtée dans la conduite se remet en mouvement en sens inverse, si elle trouve au retour tout orifice fermé, c'est-à-dire si en ce point le débit est obligatoirement nul, il y aura (sous réserve des pertes de charge) une surpression égale à la dépression consentie pour éviter la cavitation.

Or, en général cette dépression, par rapport à la pression initiale, est supérieure à la surpression acceptable dans la conduite. D'où, sauf cas exceptionnels, il sera nécessaire de limiter la valeur de la surpression.

Pour ceci il faut qu'un débit inverse puisse s'établir. Par contre, il est aussi général que, pour limiter la surpression aux valeurs acceptables, le cébi maximum inversé peut coujours ètre nettement inférieur au débit de fonctionnement normal.

Le principe est donc de laisser s'établir un débit en retour, limité au minimum comparible avec la surpression acceptable, puis ensuite de procéder à une fermeture lente (cette dernière condition ne jouant pas dans le cas du réservoir à air et de la cheminée d'équilibre).

Comme dit au début, les procécés destinés à limiter les surpressions étant conditionnés par ceux adoptés pour limiter les dépressions, c'est en fonction de ces derniers que nous en ferons lexamen.

Les dispositifs suivants résolvent simuitanément le problème de la dépression et cie la surpression sur le troncon de conduite qu'ils protègent.

\section{Ce sont :}

Les réservoir à air, cheminée d'équilibre et réservoir surélevé.

Suivant le principe général, le débit de retour est freiné por une perte de charge, entre iuyauierie d'une part, et réservoir ou cheminée d'autre part, toujours beaucoup plus grande que la perte à l'aller, ce qui est réalisé soit par des ajutages spécioux ou clapets percés, soit par des dispositifs à pertes dissymétriques.

La valeur de l'étranglement est à déterminer dans chaque cas.

Les soupapes s'ouvrant à la dépression doivent, comme déjà dit, laisser au retour de l'eau une ouverture restreinte, qui se ferme ensuite frogressivement.

Les réservoirs à eau au départ de la station devront être munis d'une soupape analogue aux précédentes.

Les clapets percés dont le fonctionnement sur conduite a été décrit précédemment. Pour limiter la surpression, I'orifice doit être tel que le débit de retour soit suffisamment faible pour être arrêté instantanément sans surpression anormale.

Par contre, les cas d'alimentation du refoulement par l'aspiration et d'arrêt sur volant imposent à la station, pour la limitation des surpressions, les dispositifs indépendants suivants.

\section{Deux cas sont à considérer :}

\section{A) LE DEVIRAGE DE LA POMPE EST ACCEPTE.}

Dans ce cas, à l'inversion de courant, l'eau revient en arrière, d̀ travers la pompe, fonction.. nant alors en turbine centripète. De préférence, pour éviter une perte d'eau importante, et un fonctionnement de la pompe à l'emballement sous pleine chute, la section laissée libre au retour est réduite, afin de limiter le débit inversé à la valeur minimum compatible avec la surpression acceptable, débit à déterminer dans chaque cas.

Cette dernière section sera fermée ensuite lentement comme pour un arrêt de turbine.

Lo fermeture sur la conduite, au départ de la pompe, se fera donc en général en deux iemps :

l'un rapide, obstruant la majeure portie de la section, en un temps correspondant à celui mis par l'eau à ralentir et s'arrêter,

l'autre lent, achevant la fermeture totale, sur le courant inversé.

Les solutions possibles qui devront être normalement réalisées avec des appareils fonctionriant sans électricité sont les suivantes:

a) Les deux temps de la fermeture sont réalisés par le même appareil, placé sur la canalisation de refoulement de la pompe comme:

- vanne à commande hydraulique,

- papillon, 
actionné dès la disjonction, avec mancuvre de fermeture répondant à la loi imposée;

b) Les deux temps sont réalisés par des organes distincts.

Dans ce cas, un premier organe, papillon ou clapet C (fig. 4) placé sur la canalisation principale en assure la fermeture rapide et totale.

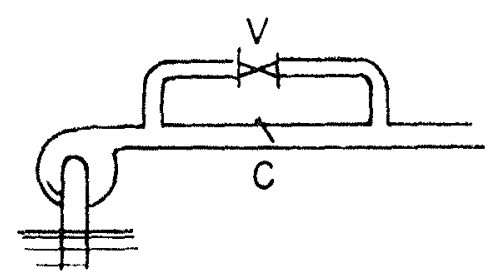

Fig. 4

Mais il est by-passé par une conduite de section réduite sur laquelle un autre appareil $V$, vanne à commande hydraulique, papillon, ouvert ou moment du démarrage, se ferme lentement ¿) la disjonction.

\section{B) LE DEVIRAGE DE LA POMPE DOIT ETRE EVITE.}

Pendant l'arrêt du groupe un appareil à fermeture rapide (papillon ou clapet C - fig. 5) doit obstruer totalement la canalisation de refoulement.

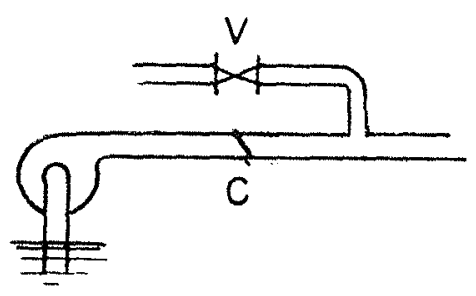

Fig. 5

Simultanément une décharge de section réduite $V$ doit s'ouvrir. Elle se referme ensuite lentement.

Le problème est anologue à celui du déchargeur de turbine. Cette solution est certainement la plus délicate à réaliser mécaniquement.

A noter que dans le cas d'alimentation à l'arrêt du refoulement par l'aspiration, il peut suffire de dashpoter à la fermeture le clapet du by-pass, la pompe étant isolée dès l'inversion de courant par un clapet ou papillon.

\section{DISCUSSION}

$M$. le Président remercie $M$. BERGERON.

Répondont à une question de M. ESCANDE, M. BERGEROr: revient sur le p'oblème de la dépression consécutive d'arrê: d'une pompe at drcule comme suit ies divers cas possibles :

Comme dola dit, frequmment dans les installations de pompage $\frac{a V_{0}}{g}$ est superiour à to hauteur manométrique totale, si bien qu'un arré imstantanes sans protection picvoquerait la cavitation gentraliste dons la conduite. C'est le cas de la fig. I.

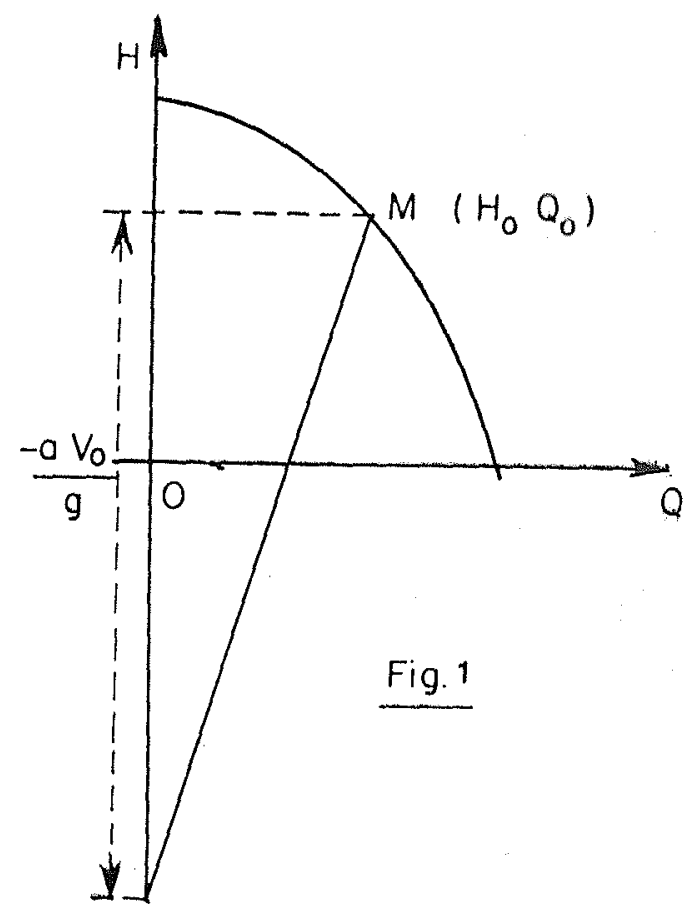

Cependant, Jorsque la houteur manométrique est grande, par exemple $800 \mathrm{~m}$., ou lorsque la vitesse initiole dans la conduite est très faible, $\frac{a \text { Vo }}{g}$ est inférieur à la hauteur C'est le cos de la tig. 2

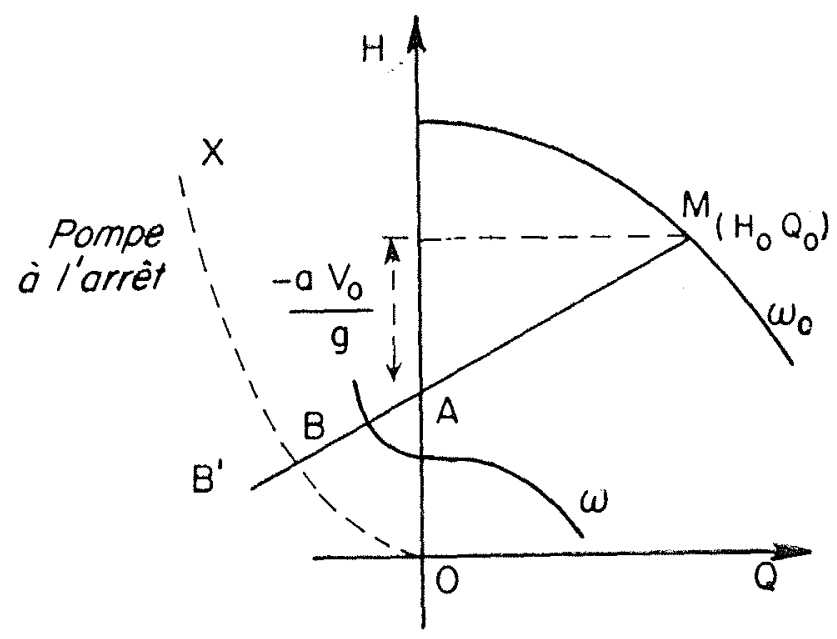

Fig. 2 
Si la pompe est munie dun clapet qui empêche le débit négatif, Ja dépression est limiée a $\frac{-a V_{0}}{g}$; mais il est exact, que s'il n'y a pas de clopet, !a dépression peut être supérieure à cette valeur.

Si dans le temps d'un aller-retour d'onde, le groupe, sous l'effet de son inertie, est passé à la vitesse (a), le point figuratif de fonctionnement sera en B (fig. 2) avec pression inférieure à $\frac{a V_{0}}{g}$. Ce point $B$ est à l'intersecion de la droite $\mathrm{MA}$ et de la caractéristique en pompe inversée, c'est-à-dire que to courant revient en arrière dans la pompe tournant encore dans le sens normal, fonctionnement toujours bruyant.

La dépression maximum-maximorum est atteinte si la pompe s'arrête, avant de repartir en sens inverse en rurbine centripète, en moins d'un aller-retour d'onde. Le point figurotif est alors en $B^{\prime}$ à l'intersection do la droite $M$ A avec la caractéristique $O X$ de la pompe à l'arrêt, qui est, en général, la caractéristique ex!rême dans les débits négatifs par rapport aux caractéristiques en pompe inversée ef en turbine centripète.

$M$. GADEN demande à $M$. BERGERON $s^{\prime}$ il $n$ 'a jamais rencontré de difficultés à construire un clapet s'ouvrant por lui-même pour parer à une surpression et si une soupaps s'ouvrant sous l'effet d'une surpression peut être construi.e avec une inertie assez faible pour effectuer sa course ossez rapidement, laisser passer un débit suffisant et limiter ia surpression.

M. BERGERON répond que les soupapes s'ouvrant sous l'effet de la dépression sont généralement employées, car elles présentent la sécurité d'être ouver'es au moment où risquerait de se produire la surpression.

Comme déjà dit, les soupapes s'ouvrant à la surpressicn devraient être sans inertie, car une fermeture de poche constitue vraisemblablement un phénomène protiquement instantoné.

En ce qui concerne les Elapets, qui ont obiliga'oirement une inertie, ils ne peuvent être emplcyés que parce que certe inertie n'est en général pas préjudiciable. En effet, le clapet se ferme au moment où le courant s'inverse, c'est-à-dire pratiquement au moment cù la dépression est maximum. Si l'inversion de courant est rapide, le clape? se ferme avec du retard et, entrainé par le couront, retombe d'ailleurs lourdement sur son siège; mais la surpression produite, s'ajoutant a une pression faible, donne une pression finale souvent très inférieure à la pression maximum admissible. Encore faut-il s'en assurer au préalable.

Il est certain qu'un clapet qui resterait accroché, pour retomber ensui:e avec un grand retard, provoquerait l'accident. Etant donné la rusticité de l'appareil il ne semble pas que ce mauvais fonctionnement soit à craindre, comme le prouve d'ailleurs l'expérience.

$M$. VIBERT indique que I'on procède actuellement en Francè à la construction ou à la reconstruction d'un grand nombre de réseaux de distribution d'eau, dans lesquels la protection des conduites de refoulemen: et autres contre les coups de bélier a été généralement négligée, le plus souvent à cause du caractère inégal que présente ce problème pour beaucoup de techniciens.

Il demande en conséquence si lej maisons spécialisées ne pourraient pas étudier et réaliger un nombre réduit de types d'appareils, simples et d'un fonctionnement sûr, tels que les réservoirs à air par exemple, dimensionnés pour protéger éventuellemen: une gamme aussi étendue que possible de réseaux ruraux ou de villes de moyenne importance.

A titre de simple indication, il souligne que pour de tels résecux, des conduites de refoulement ont des longueurs généralement comprises entre quelques centaines de mètres et $1 \mathrm{~km}$., les pressions de refoulement sont de l'ordre de $50 \mathrm{~m}$. et les débits instantanés peuvent varier de 5 à 30 litres/seconde.

Il est entendu que les réseaux plus importants ou ayant des caractéristiques nettement différentes devraieñt faire l'objet d'études spéciales.

M. BERGERON répond que dans les limites d'utilisation Envisagées, 800 à $1.000 \mathrm{~m}$; 4 d̀ 30 litres/seconde, :l apparait certain que le réservoir à air est le dispositif s'adaptant Je mieux ¿̀ une standardisation. II ne nécessite dos lo subtilité d'utilisation des autres procédés, et comme poir les caractéristiques données, il reste d'importance réduite, il doit être économiauement acceotable.

M. DUBIN demande si l'azote présente un avantage sur l'air pour l'alimentation des réservoirs.

M. PONSAR signale que l'azote utilisé dans bien des installations comportant un réservoir présente les 3 avantages suivants, classés por ordre d'importance:

- Facilité de se procurer à peu de frais l'azote en bouteilles sous $150 \mathrm{~kg} / \mathrm{cm}^{2}$. Ceci permet d'éccnomiser le prix d'un ou même deux groupes compresseurs (l'un étant souvent prévu en secours).

- Emploi d'un gaz inerte qui n'attaque pas l'intérieur du réservoir.

- Solubilité dans l'eau un peu plus faible que l'oxygène. Une seule bouteille a pu servir dans certaines installations plusieurs années consécutives.

M. DANEL signale qu'aux Etats-Unis, on vend un appareil dont l'intérieur est recouvert de caoutchiouc; par conséquent l'air n'est plus en con:act avec l'eau. En ce qui concerne les soupapes, $M$. DANEL remarque que l'inertie ne doit pas être nulle, mais doit être minime et adaptée à la limitation de la surpression désirée et à un fonctionnement sans vibration. Une soupape réglant la pression à peu près au $1 / 1.000^{\circ}$ a été étudiée au Laboratoire Dauphinois d'Hydraulique et décrite dans "La Houille Blanche».

$M$. BERGERON pense qu'une poche de vide qui se ferme proveque un arrêt: instantané.

M. DANEL observe que, dans ce cas, il y aura covitation et que l'air dissous dans l'eau se dégagera et la poche d'air ainsi formée aura le temps, en principe, de se comprimer pendant que la soupape va s'ouvrir. 